\title{
Edible bird's nest: physicochemical properties, production, and application of bioactive extracts and glycopeptides
}

\begin{abstract}
Edible bird's nest (EBN), the swiftlet's nest, has been consumed for centuries as a tonic or health food. The information upon the usage and benefits of the EBN are largely based on historical and observational reports since its discovery, until some were proven scientifically at recent times. Studies have been conducted on the major components of the EBN, i.e. glycoprotein, in attempts to elucidate the roles of the glycoprotein in terms of functionality toward health improvement. This article presents a review of the physicochemical and nutritional composition of EBN from different harvested locations and its functional properties. The conversion of EBN glycoproteins to glycopeptides via suitable hydrolysis methods to produce bioactive EBN glycopeptides was demonstrated, and it showed improvements in the functionality and nutritional values of EBN. Some applications of EBN glycopeptides into the new development of food and beverage products showed positive results, implicated to the bioavailability of the EBN glycopeptides. The combined information in this review will benefit the scientific communities, EBN manufacturers, and nutraceutical industries.
\end{abstract}

Keyword: Edible nest; Nutritional value; Bioactive glycopeptide; Functional food 
